
Informations

une revue Gallia

Rhône-Alpes | 2009

\title{
Vienne
}

Théâtre antique, rue du Cirque

\section{Tony Silvino}

\section{(2) OpenEdition \\ 1 Journals}

Édition électronique

URL : http://journals.openedition.org/adlfi/3474

ISSN : 2114-0502

Éditeur

Ministère de la culture

Référence électronique

Tony Silvino, «Vienne », ADLFI. Archéologie de la France - Informations [En ligne], Rhône-Alpes, mis en ligne le 01 mars 2009, consulté le 19 avril 2019. URL : http://journals.openedition.org/adlfi/3474

Ce document a été généré automatiquement le 19 avril 2019

(c) Ministère de la Culture et de la Communication, CNRS 


\title{
Vienne
}

\author{
Théâtre antique, rue du Cirque
}

\section{Tony Silvino}

\section{Identifiant de l'opération archéologique : 10023}

Date de l'opération : 2009 (SP)

1 Dans le cadre d'un projet d'assainissement par la ville de Vienne, une opération d'archéologie préventive a porté sur la réalisation d'une tranchée pour l'installation d'une canalisation entre la partie sud du collecteur antique, localisé dans le théâtre, et la montée Saint-Marcel, via la rue du Cirque. Malgré l'exiguïté de la tranchée et la présence de réseaux contemporains, un ensemble de vestiges gallo-romains appartenant à plusieurs états de construction a été mis au jour.

2 Le premier rassemble deux structures antérieures à la mise en place du théâtre visible actuellement. Il s'agit tout d'abord d'une maçonnerie localisée dans la partie méridionale de la fosse de scène, sur laquelle repose l'une des bases de pilier supportant le plancher de la scène. Si le mode de construction reste identique, à savoir une maçonnerie réalisée à l'aide de moellons de gneiss/micaschistes noyés dans un liant de chaux, la couleur orangée et la dureté du mortier semblent la distinguer du reste des maçonneries découvert lors de cette opération. Il est par ailleurs assez difficile de préciser son plan compte tenu de l'étroitesse de l'excavation. Quant à son orientation, elle suit de manière globale celle du théâtre. Les éléments chronologiques restent assez limités. L'orientation et la localisation de cette maçonnerie dirigent son interprétation vers une construction antérieure au théâtre qui pourrait correspondre à un premier édifice de spectacle. D'après les recherches récentes, la mise en place du théâtre est placée au début du règne de Claude et il serait surprenant qu'une ville comme Vienne n'ait pas été dotée de ce type de monument avant cette période.

3 Un second vestige mis au jour dans la rue du Cirque appartient également à une phase antérieure. Il correspond à une fondation maçonnée assez large localisée dans l'angle 
sud-ouest du théâtre. Son orientation nord-ouest - sud-est et sa localisation ne l'intègrent pas dans le plan général de l'édifice. Ses caractéristiques techniques et son tracé, qui suit la courbe du flanc de la colline de Pipet, semblent opter pour un mur de soutènement. Ce dernier supporterait ainsi une terrasse qui pourrait être liée à la construction de ce premier édifice. Si cette hypothèse paraît très séduisante, rien ne permet à l'heure actuelle de la corroborer, sans que l'on puisse pour autant l'écarter.

Le second état correspond à la construction du théâtre proprement dit avec, en premier lieu, les fondations puissantes du bâtiment de scène qui se partagent en deux parties principales de part et d'autre de la fosse de scène. Si la première est localisée à l'ouest du théâtre contre la rue du Cirque, la seconde est juxtaposée à l'ouest de l'égout nord-sud. Son profil en gradin est peut-être lié à la fosse de rideau. Les parements de ces deux fondations forment l'hyposcaenium au milieu duquel une maçonnerie rectangulaire constituait l'une des bases de pilier supportant le plancher de la scène. La présence de traces de rubéfaction contre les bords de la fosse et l'installation de gros blocs de calcaire du Midi attestent un réaménagement de la fosse, voire du théâtre, causé peut-être par un incendie. D'autre part, le mur du portique postscaenium,adossé à l'ouest du bâtiment de scène, a été localisé dans la rue du Cirque dont il suit d'ailleurs l' orientation. Son mauvais état de conservation ne permet pas de connaître sa largeur, ni sa relation avec les structures de l'esplanade suitée à l'ouest. D'autant plus qu'il existe un fort abaissement de terrain à cet emplacement. Si la largeur de l'espace de circulation est dorénavant connue, la hauteur et l'architecture du portique restent encore à définir. Concernant la datation, un remblai lié à la construction du mur a livré une quantité de céramiques certes peu conséquente, mais assez homogène, permettant de fournir un terminus fixé au milieu du I ${ }^{\text {er }}$ s. apr. J.-C. Cet élément chronologique semble rejoindre ainsi la datation claudienne proposée pour la construction de l'édifice. Le dernier élément correspond à la fondation du mur méridional de l'enceinte du monument découvert dans la rue du Cirque. Au sud de ce mur, un ensemble de vestiges reste indéterminé dans la mesure où il a été partiellement détruit par les réseaux contemporains. Il s'agit tout d'abord d'un grand bloc de molasse encadré au nord et au sud par deux maçonneries légères. Cet aménagement fonctionne avec un glacis maçonné localisé plus au sud, qui semble suivre le pendage du terrain. Localisés aux abords sud du théâtre, ces vestiges peuvent correspondre à des accès du monument. En l'absence de preuves déterminantes, la prudence s'impose quant à leur interprétation.

5 L'exploration de la fosse de la scène a permis de mettre au jour une série de remblais hétérogènes qui ont livré des lots de mobiliers très riches appartenant à l'Antiquité tardive. Cette richesse du comblement de la fosse avait déjà été repérée lors du dégagement du théâtre par J. Formigé. Le comblement est constitué par deux ensembles principaux de mobiliers. Le premier correspond a priori à des éléments d'habillage du théâtre: fûts et tambours de colonnes, plaquages de marbre, éléments du pulpitum, fragment de bas-relief, éléments de tuyauterie, etc. Or certaines pièces, comme ex-voto, indiquent eux une origine différente. En effet, il semble que des éléments appartenant à d'autres monuments périphériques aient été rapportés dans l'enceinte du théâtre destinés probablement à alimenter les fours à chaux attestés dans le secteur. Le second ensemble correspond plutôt à un dépotoir domestique caractérisé par une grande variété de mobiliers: vaisselles en céramique, en verre et métal, amphores, objets divers, monnaies, faune et restes de construction (tuiles, briques, tubuli, etc). L'expertise de ce matériel place le comblement final de la fosse à la fin du III ${ }^{\mathrm{e}} \mathrm{s}$. ou au début du siècle 
suivant. Ces niveaux sont scellés par un remblai dont la chronologie est à placer au cours $\mathrm{du} \mathrm{V}^{\mathrm{e}} \mathrm{s}$. Quant aux périodes moderne et contemporaine, elles se manifestent par un bâti constitué d'un mur de soutènement dans la rue du Cirque et de vestiges d'habitations localisés dans l'enceinte actuelle du théâtre.

6 En résumé, cette opération a permis non seulement de confirmer mais également de compléter le plan du théâtre avec chose rare quelques éléments de datation. Par ailleurs, des vestiges antérieurs à la construction du monument pourraient se rapporter à un premier édifice de spectacle. Pour finir, l'abandon du théâtre est bien attesté au début de l'Antiquité tardive, qui évolua d'un édifice de spectacle à une carrière ouverte et une zone de dépotoir.

7 Silvino Tony

INDEX

Index chronologique : Antiquité, Ier siècle apr. J.-C., IIe siècle apr. J.-C.

Thèmes : théâtre, tuile

Index géographique : Rhône-Alpes, Isère, Vienne

operation sauvetage programmé (SP)

\section{AUTEUR}

TONY SILVINO 